REVISTA INTERNACIONAL DE CIENCIAS DEL DEPORTE International Journal of Sport Science

Rev. int. cienc. deporte

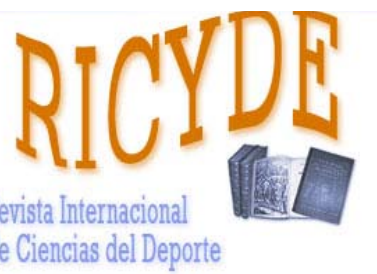

International Journal of Sport Science VOLUMEN VII - AÑO VII

Páginas:216-229 ISSN:1885-3137 No 24 - Julio - 2011

\title{
Análisis de las competencias adquiridas y utilizadas por los egresados maestros en Educación Física \\ Analysis of the skills acquired and used by graduated teachers in physical education
}

\author{
María del Carmen Campos Mesa \\ Francis Ries \\ Óscar Del Castillo Andrés \\ Facultad de Ciencias de la Educación. Universidad de Sevilla
}

\begin{abstract}
Resumen
En este artículo se plantea un análisis de las competencias que dicen haber adquirido los egresados de Magisterio de la especialidad de Educación Física durante sus estudios, y las competencias que utilizan esos mismos sujetos una vez insertos en el mercado laboral. Para ello, se diseñó un cuestionario ad hoc titulado Cuestionario sobre la inserción laboral de los maestros especialistas en Educación Física egresados en la Facultad de Ciencias de la Educación de la Universidad de Sevilla, cuyo acrónimo es E.E.F.U.S., pasándoselo a seis promociones de egresados de Magisterio $(\mathrm{N}=104)$. A los datos obtenidos se les ha realizado un análisis factorial y comparativo entre poblaciones. Podemos concluir que la competencia más valorada por los egresados en sus dos momentos de la vida, es decir, al finalizar sus estudios y una vez insertos en el mercado laboral, es aplicar el juego como recurso didáctico y contenido de enseñanza. Además, proponemos la agrupación de las competencias específicas en cuatro factores o dimensiones a trabajar con los futuros Grados en Educación Primaria con mención en Educación Física.
\end{abstract}

Palabras clave: competencias; egresados; educación física; educación superior; salidas profesionales.

\begin{abstract}
This article aims to analyse and compare the competencies which teacher-training students (Physical Education - PE) claim to have acquired during their studies and competencies which they claim to have once they have past the transition to working life, and they are already incorporated in the labour market. For that purpose, we designed a questionnaire entitled "Survey on the employment of teachers in physical education graduated from the Faculty of Education at the University of Seville (EEFUS)", and the graduated PE teachers $(\mathrm{N}=104)$ from the last six years from the University of Seville completed that specific questionnaire. We submitted the data obtained to descriptive and factorial analysis and the results inform that the competence they value most, in the two moments of their life (after graduation and once inserted in the labour market), it is to apply games as didactic material and teaching content. The analysed competencies are grouped into four factors or dimensions in order to be employed working easier with future teachers in primary education with a major in PE.
\end{abstract}

Key words: competencies; graduates; physical education; higher education; career opportunities.

Correspondencia/correspondence: María del Carmen Campos Mesa

Facultad de Ciencias de la Educación. Departamento de Educación Física. Grupo de Investigación HUM-507. Calle Pirotecnia, s/n. Sevilla 41013.

E-mail: mccampos@us.es 
Campos, M.C.; Ries, F.; Del Castillo, O. (2011). Análisis de las competencias adquiridas y utilizadas por los egresados maestros en Educación Física. Revista Internacional de Ciencias del Deporte, 24(7), $216-229$. http://www.cafyd.com/REVISTA/02405.pdf

\section{Introducción}

Cuando hablamos de inserción laboral encontramos en la literatura dos puntos de vista distintos. Por un lado, la inserción laboral como hecho puntual, es decir, conseguir un contrato laboral (Figuera y Bisquerra, 1992; Ventura, 2005), y por otro lado, la inserción laboral como un proceso o transición (Justiniano, 2006; Pérez, 1996; Rose, 1991).

Basado en estas consideraciones, entendemos la inserción laboral como un concepto complejo íntimamente relacionado con la vida de las personas y las circunstancias que las rodean. Por consiguiente, el proceso de inserción laboral implica tanto a la persona como a su contexto, refiriéndose al ser humano como unidad integral, física y mental. De este modo, se hace necesario comprender el proceso de inserción en el mundo laboral desde estos dos puntos de vista y, para su entendimiento, habría que enlazarlo con los términos de transición, empleabilidad, habilidades de empleabilidad y adaptación a un nuevo entorno por parte de los egresados de cualquier titulación (Justiniano, 2006).

Por otra parte, existe un fuerte lazo de unión entre educación y empleo, siendo la educación la mejor herramienta para el empleo. El individuo debe seleccionar la titulación universitaria que le lleve ha adquirir las competencias necesarias para poder desarrollar la profesión que desea. Así, un estudiante que decide seguir con estudios universitarios para obtener la titulación de Maestro especialidad en Educación Física o, actualmente, el Grado en Primaria con mención en Educación Física, tendrá un perfil profesional con unas competencias profesionales que le permitan desarrollar un trabajo concreto. Hasta ahora, el plan de estudio que ha guiado la titulación de Maestro especialista en Educación Física no hacía referencia a las competencias profesionales, ni al perfil profesional, ni a las capacidades profesionales (Real Decreto 1440, de 30 de agosto de 1991). Existen estudios que describen el perfil profesional y las competencias del Maestro especialista en Educación Física, como son los de la ANECA (2005a), Izquierdo (1994), Oña, Bajo, Cano, Delgado, García, y Roca (1995), Maldonado (2004), C. Romero (2004, 2009) y S. Romero (2004).

En el proceso de convergencia hacia el Espacio Europeo de Educación Superior se han producido muchos cambios, uno de ellos es el interés por definir las competencias profesionales de cada titulación. Tanto a nivel europeo como estatal se han realizado investigaciones y estudios con el objeto de definir dichas competencias. Maldonado (2004) establece una propuesta sobre el futuro título de Grado en Primaria con mención en Educación Física. Así mismo, estableció que el currículo de formación de los futuros maestros debería centrarse básicamente en tres núcleos formativos:

- Adquisición de conocimientos e instrumentos que ayuden a fundamentar la reflexión psicosocio-pedagógica del hecho educativo.

- Adquisición de conocimientos y habilidades que le permitan el desarrollo de capacidades y actitudes que faciliten el nivel de madurez personal necesaria para poder asumir las responsabilidades que le son propias.

- Creación de situaciones de enseñanza-aprendizaje que faciliten al alumnado la construcción del conocimiento que le permita en su futuro trabajo plantearse una buena reflexión desde y sobre la propia práctica. 
Campos, M.C.; Ries, F.; Del Castillo, O. (2011). Análisis de las competencias adquiridas y utilizadas por los egresados maestros en Educación Física. Revista Internacional de Ciencias del Deporte, 24(7), $216-229$. http://www.cafyd.com/REVISTA/02405.pdf

Por su parte, el Libro Blanco de la ANECA (2005a), nombra 18 competencias específicas, divididas en saber, saber hacer y competencias académicas.

Asimismo, Romero (2009), interesado en conocer cuáles son las competencias profesionales específicas del Grado en Primaria con mención en Educación Física, pregunta a los maestros que están en ejercicio, a los estudiantes y al profesorado de la titulación de la Facultad de Ciencias de la Educación de la Universidad de Granada, sobre la valoración de las mismas. La muestra fue de 346 sujetos, a los que pasó un cuestionario sobre las competencias profesionales del Maestro en Educación Física (CCPMEF). Tras las conclusiones, basándose en el análisis de las competencias, realiza una propuesta consistente en tres módulos que podrían conformar el título de Grados en Educación Primaria con mención en Educación Física:

- Módulo relacionado con los aspectos socio-culturales y disciplinares básicos de lo que se va a enseñar y de su intervención didáctica.

- Modulo de fundamentos epistemológicos y metodológicos de la Educación Física.

- Módulo de gestión, coordinación de actividades docentes y de promoción, organización y participación de actividades complementarias.

Varios trabajos demuestran que los titulados en Magisterio especialista en Educación Física obtienen porcentajes de inserción laboral altos. Así lo avalan investigaciones como la de Sánchez y Pesquero (2002) llevadas a cabo en la Facultad de Ciencias de la Educación de la Universidad Complutense de Madrid. El 80.4\% de los egresados que se encontraban trabajando correspondía a los Maestros especialistas en Educación Física. Los resultados del estudio de la ANECA (2005b), nos demuestran que los maestros tienen una tasa media de ocupación general del 68.7\%, y tres especialidades copan las primeras posiciones: Idioma (76.9\%), Educación Física (74.5\%) y Educación Especial (71.4\%).

Igualmente Romero (2005) afirma que la carrera de Magisterio especialista en Educación Física es la que más expectativas socio-profesionales ofrece, pero que, al no encontrarse completamente regulado su campo de trabajo, los egresados se encuentran perdidos, y en ocasiones, abandonan la perspectiva de la enseñanza y buscan nuevos campos de actuación profesional, como pueden ser el de entrenamiento o el de dirección de actividad física y deporte en todos sus niveles (gimnasio, creación de empresas de actividades extraescolares, actividades en la naturaleza, etc.).

Por su parte Serrano, Lera, y Contreras (2007), recuerdan que el sentido de la reforma es conciliar los títulos académicos con sus perfiles profesionales, y hacen una amplia reflexión sobre la pérdida de las especialidades que se ha producido en los títulos de Magisterio, donde sólo quedan Infantil y Primaria, con la posibilidad de realizar itinerarios-especialidad optativos, lo que no alcanza el mínimo europeo de especialidad, desconociendo, además, cómo afectará esto a los puestos laborales extramuros. La posesión de una especialidad añadida al perfil de maestrogeneralista, en términos de inserción laboral, ha venido siendo una ventaja competitiva del título.

Teniendo en cuenta las consideraciones y justificaciones descritas, el planteamiento principal del presente trabajo es el siguiente: Partiendo de la valoración que hacen los egresados de Magisterio especialistas en Educación Física de la Universidad de Sevilla, de las competencias adquiridas durante sus estudios universitarios y la valoración que hacen del uso de las mismas una vez insertos en el mercado laboral, ¿podríamos establecer las que son más importantes para ellos y los módulos de aprendizaje que deberían conformar el Grado en Primaria con mención en 
Campos, M.C.; Ries, F.; Del Castillo, O. (2011). Análisis de las competencias adquiridas y utilizadas por los egresados maestros en Educación Física. Revista Internacional de Ciencias del Deporte, 24(7), $216-229$. http://www.cafyd.com/REVISTA/02405.pdf

Educación Física? Los objetivos que nos proponemos alcanzar en el presente trabajo se relacionan a continuación:

- Identificar las diferencias detectadas por los egresados en las competencias adquiridas tras finalizar sus estudios universitarios y en el uso de las mismas una vez insertos en el mercado laboral.

- Conocer las competencias más valoradas por los egresados de Magisterio de la especialidad de Educación Física, con el objeto de poder establecer los módulos que deberían conformar la titulación de Grado en Primaria con mención en Educación Física.

\section{Método}

\section{Participantes}

La muestra del estudio está compuesta por un total de 104 sujetos, 72 hombres (69.2\%) y 32 mujeres (30.8\%), egresados de Magisterio de la especialidad de Educación Física de la Facultad de Ciencias de la Educación de la Universidad de Sevilla, concretamente de seis promociones correspondientes a los cursos académicos 1998/99 hasta 2003/04, y habiéndose graduado en la convocatoria de junio de dichos años. Con respecto al proceso de selección de la muestra, los datos de la población fueron facilitados por la secretaría de la Facultad a través de un listado de estas seis promociones de egresados. Con los datos obtenidos pudimos realizar un contacto telefónico con 292 sujetos de donde obtuvimos finalmente un marco de 146 sujetos. Los 146 sujetos de la muestra suponen una tasa de respuesta del 70.54\% $(N=104)$.

La elección de estas seis promociones se debe a una característica que cumplen todas, la de llevar como mínimo tres años diplomados y por tanto, en posesión del título. Esto se fundamenta en el trabajo realizado por Teichler (2003), quien nos indica que las encuestas realizadas a egresados un año después de acabar la carrera o incluso antes proporcionan una imagen incompleta. En el Catálogo de Indicadores del Consejo de Coordinación Universitaria (Raga, 2003), propone como un indicador para obtener el porcentaje de titulados que al acabar los estudios están empleados en un trabajo que consideran satisfactorio, un mínimo de tres años después de terminar sus estudios.

\section{Instrumento}

Se diseñó un cuestionario ad hoc para la investigación titulado Cuestionario sobre la inserción laboral de los maestros especialistas en Educación Física egresados en la Facultad de Ciencias de la Educación de la Universidad de Sevilla, cuyo acrónimo es EEFUS, en formato html, pudiendo acceder al mismo a través de una dirección Web.

La validez de contenido consistió en una revisión exhaustiva del cuestionario por tres expertos de reconocido prestigio, un Catedrático de Universidad, un Técnico de la Junta de Andalucía y un experto en Inserción Laboral, cada uno de ellos vinculado a diferentes instituciones. La validez didáctica fue estimada por tres egresados Maestros especialistas en Educación Física, de diferentes ámbitos laborales y la validez de constructo se comprobó a través del análisis factorial para las preguntas 14 y 37 referidas a las competencias profesionales consideradas en el cuestionario. También se constató la fiabilidad del cuestionario a través de los diferentes estudios pretest, así como calculando el Alfa de Cronbach, con el paquete estadístico SPSS 15.0, obteniéndose un coeficiente de .797. 
Campos, M.C.; Ries, F.; Del Castillo, O. (2011). Análisis de las competencias adquiridas y utilizadas por los egresados maestros en Educación Física. Revista Internacional de Ciencias del Deporte, 24(7), $216-229$. http://www.cafyd.com/REVISTA/02405.pdf

El estudio pretest del cuestionario consistió en una prueba control y una prueba piloto. La prueba control se realizó con un grupo de egresados de Magisterio de la Universidad de Sevilla que se encontraban cursando el segundo ciclo de la Licenciatura en Ciencias de la Actividad Física y el Deporte. El cuestionario se pasó a todos los encuestados a la vez, en un aula perteneciente a las instalaciones de la Universidad de Sevilla, utilizando como formato el papel y con la presencia del investigador. En la prueba control también se aplicó la observación no estructurada. De esta forma se pudo mantener un diálogo directo con el encuestado donde se detectaron la claridad de las preguntas, el interés de los distintos ítems, así como otras alternativas de mejora del mismo. El grupo piloto estaba formado por 20 sujetos con las mismas características de la muestra de la investigación, utilizando el mismo diseño, formato y medio que se utilizaría en la recogida de datos. Esta prueba piloto nos permitió, apoyándonos en las indicaciones planteadas por Hernández; Fernández, y Baptista (2000) entre otros, lo siguiente: modificar aspectos como la redacción de las preguntas, de forma más sutil las referidas al apartado de información personal, con respecto a las competencias se realizaron cambios de conceptos utilizando un vocabulario menos técnico y más entendible. También nos permitió verificar los aspectos del protocolo de procedimiento como el funcionamiento a través de la Web para la recogida de datos. Esta validación del cuestionario nos permitió mantener la estructura inicial del mismo sin la necesidad de eliminar ninguno de sus ítems.

El cuestionario EEFUS está formado por 63 ítems distribuidos en seis bloques, donde se recogen dimensiones con respecto al historial académico previo a los estudios universitarios, la carrera universitaria, la valoración de la formación universitaria recibida, la formación continua, la vida laboral y la información personal. El bloque referido a la vida laboral se divide a su vez en dos, uno sobre el primer trabajo después de la graduación en Magisterio especialista en Educación Física (más de 6 meses de duración) y otro sobre la situación profesional actual. En esta investigación expondremos los resultados y el análisis de dos preguntas del EEFUS, concretamente los ítems 14 y 37. El ítem 14 está dentro de la valoración de la formación universitaria recibida, y hace referencia a la valoración que hace el egresado tras finalizar sus estudios de la adquisición que tiene de 17 competencias, elaboradas realizando una adaptación de las competencias específicas en Educación Física descritas en el Libro Blanco de la ANECA (2005a), las contempladas en los títulos de maestro de las propuestas para las Universidades Andaluzas (S. Romero, 2004) y las de Romero (2007).

El ítem 37 hace referencia al primer trabajo después de la graduación en Magisterio especialista en Educación Física (más de 6 meses de duración), dentro del bloque de su vida laboral, el sujeto valora el uso que hace de esas mismas 17 competencias en su primer trabajo tras graduarse. Para ambas preguntas se utiliza una escala de tipo Likert de 5 opciones ( 1 = nada; 5 = mucho).

\section{Procedimiento}

El proceso de recogida de datos constó de cuatro fases, consistiendo la primera en un contacto telefónico directamente con los egresados con objeto de localizar los correos electrónicos de los mismos, dato imprescindible para hacerles llegar la página Web donde podían acceder al EEFUS. La segunda fase consistió en enviar por correo electrónico al egresado la presentación de la investigación y el acceso a la página Web para contestar el cuestionario. En la tercera fase se envió un segundo correo electrónico de recordatorio, a fin de intentar recoger el mayor número de datos posibles. Y la cuarta y última fase consistió en el volcado de datos de forma automática en una base de datos de Microsoft Access. En esta base de datos quedaron recogidos, por orden 
Campos, M.C.; Ries, F.; Del Castillo, O. (2011). Análisis de las competencias adquiridas y utilizadas por los egresados maestros en Educación Física. Revista Internacional de Ciencias del Deporte, 24(7), $216-229$. http://www.cafyd.com/REVISTA/02405.pdf

temporal, todos los datos de los cuestionarios de cada uno de los egresados participantes y en ningún caso se reflejaron los datos identificativos del mismo. Antes de realizar el análisis estadístico, se revisó el volcado de datos para comprobar que no existían errores.

Análisis de datos

Para el análisis de datos se realizó un análisis factorial y un análisis inferencial, denominado estudio comparativo entre poblaciones, que fue posible en nuestra investigación por pasar el cuestionario a una misma muestra pero realizando preguntas de varios momentos de sus vidas, para lo cual se utilizó el paquete estadístico SPSS v.15. para Windows.

\section{Resultados}

$\mathrm{Al}$ analizar los datos del análisis inferencial basado en el contraste de hipótesis utilizando el test Kolmogorov-Smirnov y el Test de Shapiro-Wilk, para hacer la prueba de bondad de ajuste, constatamos que los datos no cumplen el supuesto de normalidad, lo que nos lleva a utilizar pruebas no paramétricas, en concreto la de Wilcoxon, tras la cual obtenemos la tabla 1. 
Campos, M.C.; Ries, F.; Del Castillo, O. (2011). Análisis de las competencias adquiridas y utilizadas por los egresados maestros en Educación Física. Revista Internacional de Ciencias del Deporte, 24(7), $216-229$. http://www.cafyd.com/REVISTA/02405.pdf

Tabla 1. Estudio comparativo entre poblaciones $(N=104)$.

\begin{tabular}{|c|c|c|c|c|c|}
\hline \multirow[b]{2}{*}{$\begin{array}{l}\text { Competencias específicas del diplomado en } \\
\text { magisterio especialista en educación física }\end{array}$} & \multicolumn{2}{|c|}{$\begin{array}{l}\text { Valoración de los } \\
\text { egresados al finalizar } \\
\text { la carrera }\end{array}$} & \multicolumn{2}{|c|}{$\begin{array}{l}\text { Valoración de los egresados } \\
\text { de las competencias, del uso } \\
\text { que le dan en sus primeros } \\
\text { trabajos }\end{array}$} & \multirow[b]{2}{*}{$\mathrm{p}$} \\
\hline & M & DT & M & DT & \\
\hline Dominar la programación del área de EF & 2.91 & 1.12 & 2.23 & 1.40 & .00 \\
\hline Aplicar estructura curricular EF & 2.61 & .97 & 2.10 & 1.33 & .00 \\
\hline $\begin{array}{l}\text { Aplicar juego recurso didáctico y contenido de } \\
\text { enseñanza }\end{array}$ & 3.66 & .98 & 3.06 & 1.65 & .00 \\
\hline $\begin{array}{l}\text { Conocer el desarrollo psicomotor del niño e } \\
\text { intervención educativa }\end{array}$ & 3.17 & .81 & 2.71 & 1.51 & .00 \\
\hline $\begin{array}{l}\text { Conocer los fundamentos biológicos y } \\
\text { fisiológicos del cuerpo humano }\end{array}$ & 3.38 & .89 & 2.65 & 1.44 & .00 \\
\hline $\begin{array}{l}\text { Conocer los procesos de adaptación del } \\
\text { ejercicio físico }\end{array}$ & 3.31 & .90 & 2.66 & 1.45 & .00 \\
\hline $\begin{array}{l}\text { Promover hábitos de salud, higiene y } \\
\text { alimentación }\end{array}$ & 3.40 & 1.03 & 3.01 & 1.50 & .00 \\
\hline Trabajar capacidades físicas & 3.46 & .91 & 2.76 & 1.53 & .00 \\
\hline Trabajar iniciación deportiva & 3.49 & .97 & 2.76 & 1.57 & .00 \\
\hline Trabajar expresión corporal & 3.50 & 1.02 & 2.40 & 1.44 & .00 \\
\hline $\begin{array}{l}\text { Relacionar la actividad física con las distintas } \\
\text { áreas de infantil y primaria }\end{array}$ & 2.81 & .98 & 2.28 & 1.48 & .00 \\
\hline $\begin{array}{l}\text { Detectar dificultades anatómico-funcionales, } \\
\text { cognitivas y de relación social }\end{array}$ & 2.62 & .91 & 2.41 & 1.35 & .06 \\
\hline $\begin{array}{l}\text { Saber aplicar técnicas de actividades físicas en } \\
\text { medio natural }\end{array}$ & 3.39 & .96 & 2.52 & 1.41 & .00 \\
\hline $\begin{array}{l}\text { Establecer las bases de utilización del tiempo } \\
\text { libre }\end{array}$ & 3.08 & .95 & 2.48 & 1.50 & .00 \\
\hline $\begin{array}{l}\text { Orientar y supervisar actividades } \\
\text { extraescolares }\end{array}$ & 2.84 & 1.08 & 2.47 & 1.51 & .03 \\
\hline $\begin{array}{l}\text { Aplicar las tecnologías de la Información y } \\
\text { Comunicación }\end{array}$ & 2.46 & .98 & 2.13 & 1.33 & .02 \\
\hline Trabajar imagen y cuerpo & 3.02 & .84 & 2.45 & 1.45 & .00 \\
\hline
\end{tabular}

Nota: $M=$ Media; $D T=$ desviación típica; $p<0.05$ 
Campos, M.C.; Ries, F.; Del Castillo, O. (2011). Análisis de las competencias adquiridas y utilizadas por los egresados maestros en Educación Física. Revista Internacional de Ciencias del Deporte, 24(7), $216-229$. http://www.cafyd.com/REVISTA/02405.pdf

Atendiendo a los resultados expuestos en la tabla 1 podemos apreciar que las medias $(M)$ obtenidas correspondientes a la valoración de las competencias que hacen los egresados al finalizar su carrera universitaria, oscilan entre 2.46 y 3.66. Por el contrario, en la valoración que hacen del uso de las mismas en sus primeros trabajos, las medias oscilan entre 2.01 y 3.06. La media del promedio sobre la valoración de las competencias tras finalizar sus estudios universitarios, es de 3.12. Por tanto, las competencias más valoradas son "Aplicar juego, recurso didáctico y contenido de enseñanza” (3.66); “Trabajar la expresión corporal” (3.50), y "Trabajar iniciación deportiva” (3.49). Por el contrario, la media del promedio para la valoración que hacen los egresados del uso de las mismas en sus trabajos es de 2.53. En este caso las competencias más valoradas son "Aplicar juego, recurso didáctico y contenido de enseñanza" (3.06); "Promover hábitos de salud, higiene y alimentación” (3.01); “Trabajar iniciación deportiva” (2.76) y “Trabajar capacidades físicas” (2.76).

Los egresados coinciden con respecto a la competencia que más valoran de las 17 en "Aplicar juego, recurso didáctico y contenido de enseñanza”, estando este valor por encima de la media del promedio, en ambos casos.

Existen diferencias significativas $(p<0.05)$ de 16 de los 17 ítems contemplados: el único ítem que obtiene un valor superior a .05 es el referido a la competencia "Detectar dificultades anatómico-funcionales, cognitivas y de relación social”, para el que no existen diferencias significativas, y en ambos momentos de sus vidas los egresados, tras finalizar sus estudios $(M=$ 3.12) y un 2.41 de uso en su primer trabajo $(M=2.53)$, lo valoran por debajo de la media de los promedios de todas las competencias valoradas con un 2.62.

\section{Análisis factorial}

Los datos obtenidos de las preguntas 14 y 37 se han sometido a un análisis factorial exploratorio, con el objeto de sintetizar en factores la relación existente entre ellos, al igual que ha sido calculado el índice de fiabilidad de los mismos en ambos momentos de la vida preguntados a los egresados. Se ha empleado el coeficiente alfa de Cronbach, obteniéndose un coeficiente de .915, cuando el egresado valora las competencias al finalizar sus estudios y de .969 cuando valora las mismas competencias en su primer trabajo.

En primer lugar, presentamos el análisis factorial de la primera pregunta (Ítem 14), donde el egresado valora las 17 competencias tras finalizar sus estudios. Comprobamos que es útil aplicar técnicas multivariantes como el análisis factorial, obteniendo un KMO significativo de .868, proporcionando un Chi-cuadrado de 928.664 y una significación de $p=.000$.

Tras realizar la extracción de componentes, podemos observar que cuatro de ellos tienen un autovalor superior a la unidad, lo que nos indica que van a ser extraídos cuatro componentes que explican el $67.87 \%$ de la varianza. El método de extracción utilizado ha sido el de componentes principales. Con objeto de ver con más claridad la correlación entre las 17 competencias, utilizamos la matriz de componentes rotados Varimax, presentados en la tabla 2. 
Campos, M.C.; Ries, F.; Del Castillo, O. (2011). Análisis de las competencias adquiridas y utilizadas por los egresados maestros en Educación Física. Revista Internacional de Ciencias del Deporte, 24(7), $216-229$. http://www.cafyd.com/REVISTA/02405.pdf

Tabla 2. Matriz de componentes rotados de las competencias valoradas tras finalizar la carrera

\begin{tabular}{|c|c|c|c|c|}
\hline & \multicolumn{4}{|c|}{ Componente } \\
\hline & 1 & 2 & 3 & 4 \\
\hline Dominar la programación del área de EF & & .762 & & \\
\hline Aplicar estructura curricular EF & & .866 & & \\
\hline Aplicar juego recurso didáctico y contenido de enseñanza & & .748 & & \\
\hline Conocer el desarrollo psicomotor del niño e intervención educativa & .539 & & & \\
\hline Conocer los fundamentos biológicos y fisiológicos del cuerpo humano & .771 & & & \\
\hline Conocer los procesos de adaptación del ejercicio físico & .855 & & & \\
\hline Promover hábitos de salud, higiene y alimentación & .511 & & & \\
\hline Trabajar capacidades físicas & .732 & & & \\
\hline Trabajar iniciación deportiva & .700 & & & \\
\hline Trabajar expresión corporal & & & .903 & \\
\hline Relacionar la actividad física con las distintas áreas de infantil y primaria & & .597 & & \\
\hline Detectar dificultades anatómico-funcionales, cognitivas y de relación social & & & & .710 \\
\hline Saber aplicar técnicas de actividades físicas en medio natural & & & .569 & \\
\hline Establecer las bases de utilización del tiempo libre & & & .845 & \\
\hline Orientar y supervisar actividades extraescolares & & & & .574 \\
\hline Aplicar las tecnologías de la Información y Comunicación & & & & .800 \\
\hline Trabajar imagen y cuerpo & & & .731 & \\
\hline
\end{tabular}

De estos resultados determinamos 4 factores:

Factor 1. Conocimientos disciplinares de educación físico-deportiva, aludiendo a un total de 6 competencias: Conocer el desarrollo psicomotor del niño e intervención educativa, Conocer los fundamentos biológicos y fisiológicos del cuerpo humano, Conocer los procesos de adaptación del ejercicio físico, Promover hábitos de salud, higiene y alimentación, Trabajar capacidades físicas y Trabajar iniciación deportiva.

Factor 2. Competencias profesionales específicas, agrupando un total de 4 competencias: Dominar la programación del área de EF, Aplicar estructura curricular Educación Física, Aplicar juego recurso didáctico y contenido de enseñanza, Relacionar la actividad física con las distintas áreas de infantil y primaria.

Factor 3. Conocimientos disciplinares de ocio y expresión corporal, aludiendo a un total de 4 competencias: Trabajar expresión corporal, Saber aplicar técnicas de actividades físicas en medio natural, Establecer las bases de utilización del tiempo libre y Trabajar imagen y cuerpo.

Factor 4. Competencias transversales aludiendo a un total de 3 competencias: Detectar dificultades anatómico-funcionales, cognitivas y de relación social, Orientar y supervisar actividades extraescolares y Aplicar las tecnologías de la Información y Comunicación. 
Campos, M.C.; Ries, F.; Del Castillo, O. (2011). Análisis de las competencias adquiridas y utilizadas por los egresados maestros en Educación Física. Revista Internacional de Ciencias del Deporte, 24(7), $216-229$. http://www.cafyd.com/REVISTA/02405.pdf

A continuación presentamos el análisis factorial de la pregunta 37. Realizamos el mismo proceso que anteriormente obteniendo una KMO significativa de .916, proporcionando un Chi-cuadrado de 1573.590 y una significación de $p=.000$.

Tras realizar la extracción de componentes, podemos observar que hay tres componentes con autovalor por encima de uno, lo que nos indica que van a ser extraídos tres factores, que explican el 76.52\% de la varianza. El método de extracción utilizado ha sido el de análisis de componentes principales. A fin de ver con más claridad la correlación entre las 17 competencias, utilizamos la matriz de componentes rotados Varimax, obteniendo la siguiente tabla 3.

Tabla 3. Matriz de componentes rotados de las competencias valoradas según el uso de las mismas en los primeros trabajos del egresado.

\begin{tabular}{|l|l|l|}
\hline & \multicolumn{2}{|l|}{ Componente 1} \\
\hline Dominar la programación del área de EF & .726 & .715 \\
\hline Aplicar estructura curricular EF & .844 & \\
\hline Aplicar juego recurso didáctico y contenido de enseñanza & .853 & \\
\hline Conocer el desarrollo psicomotor del niño e intervención educativa & .771 & \\
\hline Conocer los fundamentos biológicos y fisiológicos del cuerpo humano & .798 & \\
\hline Conocer los procesos de adaptación del ejercicio físico & .860 & \\
\hline Promover hábitos de salud, higiene y alimentación & .823 & \\
\hline Trabajar capacidades físicas & .823 & \\
\hline Trabajar iniciación deportiva & .741 \\
\hline Trabajar expresión corporal & .759 & \\
\hline Relacionar la actividad física con las distintas áreas de infantil y primaria & .834 & \\
\hline Detectar dificultades anatómico-funcionales, cognitivas y de relación social & .758 & \\
\hline Saber aplicar técnicas de actividades físicas en medio natural & .771 & .729 \\
\hline Establecer las bases de utilización del tiempo libre & .702 & \\
\hline Orientar y supervisar actividades extraescolares & .831 \\
\hline Aplicar las tecnologías de la Información y Comunicación & \\
\hline Trabajar imagen y cuerpo & & \\
\hline
\end{tabular}

De estos resultados determinamos un solo factor: Factor 1. Competencias del Profesor de Educación Física, aludiendo a las 17 competencias preguntadas a los egresados de Magisterio de la especialidad de Educación Física de la Universidad de Sevilla.

\section{Discusión}

En esta investigación hemos podido comprobar cuáles son las competencias más valoradas por los egresados de Magisterio especialistas en Educación Física de la Universidad de Sevilla, y hemos podido comparar la valoración que hacen de las mismas en dos momentos temporales de sus vidas; respectivamente al final de sus estudios valorando el conocimiento que tenían de las mismas y en el primer trabajo una vez insertos en el mercado laboral, valorando su uso. 
Campos, M.C.; Ries, F.; Del Castillo, O. (2011). Análisis de las competencias adquiridas y utilizadas por los egresados maestros en Educación Física. Revista Internacional de Ciencias del Deporte, 24(7), 216-229. http://www.cafyd.com/REVISTA/02405.pdf

En ambos casos la competencia más valorada ha sido "Aplicar juego recurso didáctico y contenido de enseñanza”. Según los egresados, esta competencia es adquirida durante la carrera y además es una de las más usadas en sus trabajos. Datos no coincidentes con el estudio de Romero (2009), donde la competencia más valorada está relacionada con los hábitos de higiene y de salud, competencia que en nuestro estudio está valorada en tercer lugar, en ambos momentos temporales. La segunda competencia más valorada por los egresados, en los dos momentos de sus vidas son el trabajo de la iniciación deportiva y de la expresión corporal, datos que sí coinciden con el estudio de Romero (2009) y con el estudio de la ANECA (2005a), donde se valora el dominio y conocimiento de la técnica, la teoría y la didáctica específica de la Educación Física para Educación Primaria.

Por lo general, los egresados valoran más las competencias cuando salen de la carrera que el uso que hacen de ellas en sus primeros trabajos. En el estudio de Romero (2009), se afirma que los egresados que se encuentran ejerciendo de maestros valoran más que los egresados recién salidos de la carrera el "saber hacer”, más cercanos a los conocimientos prácticos y didácticos. Ello es coincidente con nuestra investigación, ya que la competencia más valorada se clasifica como "saber hacer".

Se producen cambios en el mundo laboral o práctica profesional de los docentes, sobre todo por lo que la sociedad puede demandar en el Sistema Educativo como consecuencia de las modificaciones y las nuevas situaciones sociales a lo largo del tiempo (Viciana, 2000). Pudiendo influir esto en la valoración que hacen de sus competencias al salir de sus estudios y del uso que hacen una vez insertos en el mercado laboral y pasado el tiempo. Por su parte Sánchez (1993) arguye que la función docente no es la misma siempre, es cambiante y compleja, determinada por el concepto de escuela, de enseñanza y currículo que prevalece en cada momento.

Según Román y Murillo (2008), las competencias profesionales del profesorado, referidas a la preparación de la enseñanza y a la organización del proceso de trabajo en el aula, se consolidan como factores importantes que inciden en lo que aprenden y logran los estudiantes. Específicamente en el campo de la Educación Física C. Romero (2004), nos expresa lo mismo al decirnos que la formación del profesorado debe estar orientada a que los futuros docentes, a partir de unos conocimientos pedagógicos, psicológicos, didácticos, sociológicos y biológicos conozcan la Educación Física y aprendan a enseñarla.

Campos (2006) concluye en su investigación que el 54.8\% de egresados afirma que algunas de la funciones de Actividad Física y Deporte que desarrolla o ha desarrollado el Maestro Especialista en Educación Física, no son para las que son competentes y está cualificado según su titulación. Por su parte, Gallardo (2006), comenta en su estudio que los egresados que trabajan resaltan la capacidad creativa, innovadora y crítica, frente a los conocimientos técnicos relacionados con la Educación Física, competencia más valorada por los egresados que no trabajaban.

Estamos de acuerdo con Campos (2006) en que la formación de los futuros titulados de Grados en Educación Primaria con mención en Educación Física, los actuales Maestros especialistas en Educación Física, deberían tener relación con su mercado laboral, sus competencias profesionales y funciones laborales. También coincidimos con Fraile (2004) al considerar que este tipo de reflexiones sobre la práctica profesional, ya sea a través de encuesta u otro tipo, son la clave para favorecer el desarrollo profesional de los docentes. 
Campos, M.C.; Ries, F.; Del Castillo, O. (2011). Análisis de las competencias adquiridas y utilizadas por los egresados maestros en Educación Física. Revista Internacional de Ciencias del Deporte, 24(7), 216-229. http://www.cafyd.com/REVISTA/02405.pdf

\section{Conclusiones}

Con respecto al primer objetivo, de los resultados obtenidos tras el análisis de los datos, podemos señalar que las competencias más y menos valoradas coinciden por los egresados de Magisterio especialistas en Educación Física valorándolas tanto al finalizar sus estudios universitarios como del uso de las mismas una vez insertos en el mercado laboral, siendo "Aplicar juego, recurso didáctico y contenido de enseñanza” y "Trabajar iniciación deportiva” las más valoradas, y "Detectar dificultades anatómico-funcionales, cognitivas y de relación social” la menos valorada.

Con respecto al objetivo referido a conocer las competencias más valoradas por los egresados de Magisterio de la especialidad de Educación Física, con el objeto de poder establecer los módulos que deberían conformar la titulación de Grado en Primaria con mención en Educación Física, proponemos cuatro módulos, que servirán para desarrollar las competencias y definir las unidades competenciales de estos profesionales:

- Módulo relacionado con los conocimientos disciplinares de educación físico-deportiva, relacionado con conceptos específicos de la especialidad, referidos al desarrollo psicomotor del niño e intervención educativa, a los fundamentos biológicos y fisiológicos del cuerpo humano, a los procesos de adaptación al ejercicio físico, a los hábitos de salud, higiene y alimentación, trabajar las capacidades físicas y la iniciación deportiva.

- Módulo relacionado con las competencias profesionales específicas, referido a competencias más generales de los docentes, saber realizar una programación de área, aplicar la estructura curricular, aplicar recurso didáctico y contenido de enseñanza en distintas áreas.

- Módulo relacionado con los conocimientos disciplinares de ocio y expresión corporal, referida a conocimientos de expresión corporal, actividades físicas en medio natural, utilización del tiempo libre y trabajo de la imagen y el cuerpo.

- Módulo relacionado con los competencias transversales, extensibles a todas las áreas como detectar dificultades anatómico-funcionales, cognitivas y de relación social, orientar y supervisar actividades extraescolares y el trabajo de la tecnología de la Información y Comunicación.

\section{Referencias bibliográficas}

ANECA (2005a). Libro Blanco. Título de Grado en Magisterio (1). Madrid: Agencia Nacional de Evaluación de la Calidad y Acreditación.

ANECA (2005b). Libro Blanco. Título de Grado en Magisterio (2). Madrid: Agencia Nacional de Evaluación de la Calidad y Acreditación.

Campos, A. (2006). Situación profesional de las personas que trabajan en funciones de actividad física y deporte en la Comunidad Autónoma Valenciana. Tesis Doctoral. Sin publicar. Universidad de Valencia.

Carreiro da Costa, F. (2006). La enseñanza de la Educación Física ante la implantación del Espacio Europeo de Educación Superior. Educación física y deporte, 25 (2), 29-43.

Contreras, O.R.; Ruiz L.M.; Zagalaz, M.L., y Romero S. (2002). Las creencias en la formación inicial del profesor de Educación Física. Incidencias en la transformación de su pensamiento. Revista interuniversitaria de formación del profesorado, 45, 131-149. 
Campos, M.C.; Ries, F.; Del Castillo, O. (2011). Análisis de las competencias adquiridas y utilizadas por los egresados maestros en Educación Física. Revista Internacional de Ciencias del Deporte, 24(7), $216-229$. http://www.cafyd.com/REVISTA/02405.pdf

Davia, M.A. (2004). La inserción laboral de los jóvenes en la Unión Europea. Un estudio comparativo de trayectorias laborales. Madrid: Consejo Económico y Social.

De Pablos Pons, J. (2008). Análisis estratégico para la Convergencia Europea (Un estudio sobre la Universidad de Sevilla). Sevilla: Secretariado de Publicaciones.

Del Villar, F. (2009). La calidad del aprendizaje en Educación física. Una propuesta para la educación secundaria. Tándem: Didáctica de la Educación Física, 8 (29), 64-77.

Figuera, P., y Bisquerra, R. (1992). Transició i inserció: una aproximació conceptual. IV Jornades d' d'Orientació sobre educació per a la Carrera professional. A.C.O.E.P: Barcelona.

Fraile, A. (2004). El profesor de EF como investigador de su práctica. Tándem: Didáctica de la Educación Física, 15, 37- 49.

Gallardo, M.A. (2006). Evaluación de las Competencias Profesionales para la Inserción Laboral de los Maestros de Educación Física. Revista electrónico de investigación Psicoeducativa, 4 (3), 9, 469-492.

Hernández, R.; Fernández, C., y Baptista, P. (2000). Metodología de la investigación (2o ed.). México, D.F.: McGraw-Hill Interamericana.

Izquierdo, A. (1994). La calidad de la enseñanza superior y otros temas universitarios. Madrid: ICE de la Universidad Politécnica de Madrid.

Justiniano, M.D. (2006). La inserción laboral de los profesionales graduados en Ciencias de la Educación. Tesis Doctoral. Universidad de Barcelona. Sin publicar.

Maldonado, A. (2004) (Coord.). Libro blanco del Título de grado en Magisterio I y II. http://www.aneca.es/modal_eval/conver_docs_titulos.html (Consultado: 02/11/2010).

Oña, A.; Bajo, S.; Cano, C.; Delgado, M.A.; García, J., y Roca, J. (1995). Seminario andaluz sobre definición del marco laboral del profesional de la actividad Física y del Deporte. Málaga: Instituto Andaluz del Deporte.

Pérez, N. (1996). Programa de formació per a la inserció laboral. Barcelona: CEAC.

Raga, J.T. (2003). Presentación. En J. Vidal (Coord.) Métodos de análisis de la inserción laboral de los universitarios: Seminario. León: Secretariado de Publicaciones y Medios Audiovisuales. http://sid.usal.es/idocs/F8/FD07238/estudio.pdf (Consultado: 02/02/2008)

Real Decreto 1440, de 30 de agosto de 1991, por el que se establece el título universitario, oficial de Maestro, en sus diversas especialidades y las directrices generales propias de los planes de estudios conducentes a su obtención.

Román, M., y Murillo, F.J. (2008). La evaluación del desempeño docente: objeto de disputa y fuente de oportunidades en el campo educativo. Revista I beroamericana de Evaluación Educativa, 2 (1), 1-6. http://www. rinace. net/riee/numeros/vol1-num2/editorial. pdf. (Consultado: 13/06/2009)

Romero, C. (2004). Argumentos sobre la formación inicial de los docentes en educación física. Profesorado, revista de currículum y formación del profesorado, 8 (1), 1-20.

Romero, C. (2009). Definición de módulos y competencias del maestro con mención en Educación Física. Revista internacional en medicina de ciencias de la actividad física y el deporte, 9 (34), 179-200.

Romero, S. (Coord.) (2004). Proyecto de implantación del Crédito Europeo ECTS. Guía Docente de Educación Física. Granada: Universidad de Granada. 
Campos, M.C.; Ries, F.; Del Castillo, O. (2011). Análisis de las competencias adquiridas y utilizadas por los egresados maestros en Educación Física. Revista Internacional de Ciencias del Deporte, 24(7), 216-229. http://www.cafyd.com/REVISTA/02405.pdf

Romero, S. (2005). La Formación de Educación Física y Deportiva en España. Apuntes de las Jornadas sobre el año internacional de la Educación Física, 1-25. Instituto Andaluz del Deporte.

Romero, S. (2007). Los contenidos de Grado. Mención en Educación Física. En P. Palou, F.J. Ponseti, P. A Borrás, y J. Vidal. Educación Física en el Siglo XXI. Nuevas perspectivas Nuevos retos, 207-222. Palma de Mallorca: Universitat de les Illes Balears.

Rose, J. (1991). Formación y mercado de trabajo: utilización de los datos para la toma de decisiones. Formación profesional, 2, 57-61.

Sánchez, M.E., y Pesquero, E. (2002). La importancia de la formación en el proceso de inserción laboral de los maestros. Revista complutense de Educación, 13(1), 141-160.

Sánchez, M.A. (1993). La formación inicial. El currículum del nadador. Cuadernos de Pedagogía, 161, 50-54.

Serrano, J.A.; Lera, A., y Contreras, O.R. (2007). Maestros generalistas vs especialistas. Claves y discrepancias en la reforma de la formación inicial de los maestros de primaria. Revista de Educación, 344, 533-555.

Teichler, U. (2003). Aspectos metodológicos de las encuestas a graduados universitarios. Centro para la Investigación en educación Superior y Trabajo. En J. Vidal (Coord.) Métodos de análisis de la inserción laboral de los universitarios: Seminario. León: Secretariado de Publicaciones y Medios Audiovisuales. http://sid.usal.es/idocs/F8/FDO7238/estudio.pdf (Consultado: 02/02/2008)

Ventura, J. (2005). El prácticum en los estudios pedagógicos y la inserción laboral. Nuevos enfoques ante el reto europeo. Tesis Doctoral. Universidad de Barcelona. Inédito.

Viciana, J. (2000). Principales centros de atención del profesorado de Educación Física en los primeros años de profesión docente. Motricidad: revista de ciencias de la actividad física y del deporte, 6, 107-122. 